

\title{
Référence et coréférence du pronom indéfini "on"
}

Frédéric Landragin, Noalig Tanguy

\section{To cite this version:}

Frédéric Landragin, Noalig Tanguy. Référence et coréférence du pronom indéfini "on". Langages, 2014, 195, pp.99-115. halshs-01069457

\section{HAL Id: halshs-01069457 https://shs.hal.science/halshs-01069457}

Submitted on 29 Sep 2014

HAL is a multi-disciplinary open access archive for the deposit and dissemination of scientific research documents, whether they are published or not. The documents may come from teaching and research institutions in France or abroad, or from public or private research centers.
L'archive ouverte pluridisciplinaire HAL, est destinée au dépôt et à la diffusion de documents scientifiques de niveau recherche, publiés ou non, émanant des établissements d'enseignement et de recherche français ou étrangers, des laboratoires publics ou privés. 


\title{
Référence et coréférence du pronom indéfini on
}

\author{
- DRAFT - \\ Frédéric Landragin, Noalig Tanguy \\ Lattice, ENS/Université de Paris 3 Sorbonne Nouvelle
}

\section{Introduction : approche et hypothèses}

Le pronom on ne réfère qu'à des référents humains. Qu'on le considère comme un pronom personnel, un pronom indéfini, voire comme pronom personnel indéfini (Sandfeld, 1970 ; Charaudeau, 1992 ; Grevisse \& Goosse, 2002), il a ceci de particulier qu'il peut référer aussi bien à une personne précise qu'à un générique, à un collectif clairement identifiable qu'à un groupe de personnes à l'étendue et aux limites vagues ; il peut inclure ou exclure le locuteur, renvoyer aussi bien aux interlocuteurs qu'à de tierces personnes. En contexte, compte tenu de l'ensemble de ces possibilités, la référence de on est parfois ambiguë, et parfois sousdéterminée (Fuchs, 1996) : le lecteur ou l'interlocuteur détermine facilement l'inclusion ou non de quelques personnes saillantes telles que les interlocuteurs, mais détermine plus difficilement l'inclusion ou non de référents supplémentaires.

On est en quelque sorte la forme de référence qui correspond le mieux à la notion de " groupe flou », modélisation retenue dans le projet MC4 (Schnedecker \& Landragin, ce volume; Mélanie-Becquet \& Landragin, ce volume) pour rendre compte des référents pluriels, à partir du moment où on ne sait pas exactement combien d'individus sont impliqués, à partir du moment où on est incapable de dire qui fait exactement partie du groupe de référents et qui n'en fait pas partie (Landragin, 2011).

Nous faisons ainsi l'hypothèse que l'interprétation du pronom on est souvent sousspécifiée, qu'il s'agit de l'expression référentielle préférentiellement associée à un groupe de personnes aux frontières floues. C'est la première hypothèse que cet article vise à développer.

Par ailleurs, on est probablement aussi la forme de référence qui contraste le mieux " chaîne de référence " et "chaîne anaphorique 》. Une succession de on coréférentiels conduit à considérer une chaîne de référence, mais en aucun cas une chaîne anaphorique. Etant un pronom personnel, on n'est jamais anaphorique : il recrée sa référence au fur et à mesure de ses apparitions, à l'instar des pronoms de première et de deuxième personne. Compte tenu de ce fait et de la sous-spécification référentielle fréquente de on, se pose la question de la nature des chaînes de référence incluant des occurrences de on: peut-on vraiment parler de coréférence au sens strict? Les alternances de on inclusif et de nous sontelles coréférentielles au sens strict? Quelles sont les contraintes discursives opérant sur les successions de on?

Notre deuxième hypothèse est qu'au niveau intra-phrastique, la référence de on dépend des autres références présentes dans la phrase, la coréférence locale étant privilégiée, et qu'au niveau extra-phrastique, on, en recouvrant de manière vague (ou " floue ») des groupes de référents, est un marqueur de cohésion et de cohérence.

Ce sont ces deux hypothèses quant aux particularités de on qui nous ont amenés à étudier ses occurrences en corpus, avec les préoccupations du projet MC4 concernant les annotations des phénomènes de référence et de coréférence. Au-delà d'une étude sur une forme linguistique particulière, notre objectif est aussi de constituer - ou du moins de 
commencer à constituer - un corpus d'étude sur la référence floue. Dans cet article, nous explorons ainsi quelques facettes de la référence et de la coréférence de on, en partant d'une liste de contraintes et d'hypothèses, et en confrontant cette liste avec des observations et des analyses réalisées sur un corpus comprenant essentiellement la pièce de théâtre Juste la fin du monde de Jean-Luc Lagarce et la nouvelle L'occupation des sols de Jean Echenoz. Nos observations et conclusions resteront à mettre en perspective avec un corpus de plus grande taille et comprenant un plus grand nombre de genres textuels différents.

\section{A propos de on}

Classé parfois dans la catégorie des pronoms personnels (Charaudeau, 1992), parfois dans celle des pronoms indéfinis (Sandfeld, 1970 ; Grevisse \& Goosse, 2002), parfois également comme pronom personnel indéfini (TLFI), ou encore comme pronom impersonnel (Cabredo Hofherr, 2008 ; Creissels, 2011), avec selon les époques des allers-retours entre les catégories - cf. l'article de Nathalie Fournier et l'article de Francine Mazière dans (Danon-Boileau \& Morel, 1994) - on pose toujours des problèmes de catégorisation, problèmes qui reflètent sa complexité d'emploi. L'important, c'est d'une part qu'il peut jouer un rôle de pronom personnel, valant pour je, $t u, i l, n o u s, v o u s, i l s$, d'autre part qu'il peut jouer un rôle de pronom indéfini, valant soit pour quelqu'un, des gens (emploi épisodique), soit pour chacun, les gens (emploi générique), et ce en suivant un ensemble de contraintes syntaxiques et sémantiques qui lui sont propres.

\subsection{Contraintes d'emploi}

L'emploi de on est soumis à quelques contraintes. Il est notamment toujours nominal et sujet. C'est un pronom clitique : pas d'accentuation prosodique, pas de modifieur, contrainte sur la position par rapport au verbe, inversion clitique du sujet possible (" peut-on »), etc. Le verbe conjugué doit être au singulier - ce qui n'a pas toujours été le cas (Rey, 2012, page 2325) mais les adjectifs et participes passés ne satisfont pas forcément cette contrainte. On le voit dans (1) et (2), où on réfère respectivement à un groupe de femmes (à l'écrit) et à une femme générique (à l'oral). Ainsi, la morphologie peut donner des indices pour aider à la résolution de la référence de on. Le régime réfléchi est se ou soi (3). Quand il faut exprimer un pronom personnel - à l'exception du réfléchi - renvoyant à on, nous (1) ou vous (4) est indispensable. Quand il faut reprendre le référent de on, pour établir une relation de coréférence, il est nécessaire de répéter on (5).

(1) Cela nous rend service et on n'est pas toujours obligées de demander aux autres (Jean-Luc Lagarce, Juste la fin du monde, page 24 dans l'édition «les Solitaires intempestifs », 2000)

(2) Tu comprends qu'on est prise au piège ? (Marie Cardinal, exemple extrait via une requête de Frantext, R205, page 136)

(3) On pouvait se baigner par places (Guy de Maupassant, La mère sauvage, début)

(4) On n'ose plus se demander si cela vous plaît (Marcel Proust, exemple cité par Grevisse \& Goosse, 2002, page 965)

(5) On attrape un rhume si on (*il) ne fait pas attention (Cabredo Hofherr, 2008, page 39)

(6) On retourna son portrait contre le mur et $\varnothing$ fit défense de prononcer son nom (JeanPaul Sartre, exemple cité par Grevisse \& Goosse, 2002, page 965) 
D'autres principes, qui ont été considérés comme des contraintes à une époque ou par certains grammairiens, ne s'appliquent pas vraiment. C'est le cas de l'usage de la forme l'on, considéré parfois comme préférentiel derrière certains mots et défendu derrière d'autres, qui s'avère de fait arbitraire (Sandfeld, 1970, page 330). C'est encore le cas de la répétition devant les verbes coordonnés, classiquement obligatoire, et qui peut de fait s'éviter (6).

\subsection{Aspects sémantiques}

On s'interprète de multiples manières. Comme un pronom personnel, il réfère à une ou plusieurs personnes, avec une lecture spécifique aussi bien que générique. Les composants de la phrase dans laquelle il apparaît peuvent contraindre ou du moins favoriser une lecture plutôt qu'une autre. Dans ses études des lectures possibles de on, Patricia Cabredo Hofherr montre par exemple qu'en fonction du prédicat ou encore de la portée par rapport aux adverbes, une lecture épisodique de on est possible (7), avec un comportement différent de celui des pronoms indéfinis tels que quelqu'un (8) (9). Denis Creissels parle de lecture gnomique pour faire une distinction entre les on génériques et les on qui interviennent dans des phrases exprimant des généralisations (10). Du fait de son indétermination, on peut contribuer à l'ambiguïté de certaines phrases. Dans son étude de l'ambiguïté en français, Catherine Fuchs ne présente pas de section spécifique sur on, mais celui-ci apparaît dans quelques exemples où il serait plus difficile de garder l'ambiguïté avec un autre pronom ou une autre expression. C'est le cas de l'exemple (11), où l'ambiguïté est présentée comme celle du procès (Fuchs, 1996, page 157), mais pourrait être interprétée comme résultant de la combinaison de plusieurs valeurs potentielles pour on avec plusieurs valeurs potentielles pour la construction ne pas fumer ici : alternative entre une valeur constative ( ici il n'y a pas de fumeur ») et une valeur déontique (« il est interdit de fumer ici »).

(7) Hier on m'a volé mon vélo (Cabredo Hofherr, 2008, page 36)

(8) On m'a volé mon vélo deux fois (deux vols, a priori deux voleurs, éventuellement le même) - quelqu'un m'a volé mon vélo deux fois (deux vols, forcément le même voleur) (Cabredo Hofherr, 2008, page 38)

(9) On t'a appelé plusieurs fois (mais la personne en question n'a pas pu attendre / mais ce n'était pas la même personne) - quelqu'un t'a appelé plusieurs fois (forcément la même personne) (Creissels, 2011, page 8)

(10) En Italie, on sait préparer les pâtes (Creissels, 2011, page 12)

(11) Ici on ne fume pas (Fuchs, 1996, page 158)

Le contexte phrastique peut cependant apporter certaines indications qui valent comme contraintes référentielles. Le genre du référent de on peut être identifié, soit par la morphologie - accord visible à l'écrit, comme en (1), ou audible à l'oral, comme en (2) - soit par des aspects sémantiques. En (12), la sémantique de la phrase impose a priori le genre masculin pour l'attribution de référents à on.

(12) On demande bien à une jeune fille d'être votre femme (Myriam Harry, exemple cité par Sandfeld, 1970, page 331)

Quand on rencontre me ou nous, il rend toute référence inclusive impossible : le locuteur de (13) est exclu de la référence de on, qui prend un sens exclusif, $t u$, il, vous ou ils. Claire Blanche-Benveniste l'a montré en passant en revue les emplois de on dans un corpus de grande taille, et en a fait une règle qu'elle a nommée "règle de schizophrénie partielle »: « une personne complexe ne peut pas se combiner syntagmatiquement avec des personnes 
dont elle est constituée »(Blanche-Benveniste, 1987, page 19). Des contre-exemples sont cependant possibles, y compris dans notre corpus de taille réduite, et c'est pourquoi nous mentionnons cette règle dans les aspects sémantiques plutôt que syntaxiques. Dans (14), il est par exemple difficile de dire si on exclut le locuteur ou si, justement, il vaut pour lui, un peu comme «je me comprends» (bien que, dans ce cas, " on se comprend » serait peut-être préférable).

(13) Au lieu de me parler, on s'adresse à ma canne (Frantext, R157, page 189)

(14) On me comprend (Juste la fin du monde, page 17)

Quand on fait partie d'une expression quasi-figée qui constitue une greffe ou un couplage, comme dans (15) - que l'on peut aussi interpréter comme " il avait une sorte de tonsure là » on peut considérer qu'il est inutile d'essayer d'attribuer un référent à on. On aurait dit exprime l'incertitude, de même que on ne sait où signifie quelque part; on ne sait quand signifie un jour, et ainsi de suite, sans faire intervenir de référent humain particulier ni de phénomène référentiel notable.

(15) Il avait on aurait dit une tonsure là (Deulofeu, 1989, page 129)

Une chaîne de référence comprenant un on peut comprendre des nous et des vous, de même que toute autre expression référentielle. A l'oral, la construction "nous, on » est d'ailleurs courante. D'une manière générale, les alternances de on et de nous sont fréquentes et ont fait l'objet de plusieurs études. Les critères souvent avancés pour expliquer l'utilisation d'un on au milieu de nous sont les suivants : on est plus facile à utiliser quand on doit mettre un réflexif (« on se regarde » évite la répétition et la lourdeur de «nous nous regardons »); la conjugaison des verbes est plus simple et plus courte à la troisième personne du singulier qu'à la première personne du pluriel; on n'est pas forcément le premier terme qui apparaît et n'a donc pas le rôle de fixer la référence qui revient à nous, comme dans «nous, on ».

Une véritable alternance de on et de nous n'apparaît cependant que quand nous est sujet et non détaché : «nous, nous » est possible, mais pas * «on, on », donc «nous, on » ne peut pas être considéré comme une alternance sur un pied d'égalité. Par contre, (16), (17) et (18) présentent une telle alternance, il est vrai pas aussi fréquente que celle du «nous, on ». Si (17) et (18) vont dans le sens des arguments précités avec des réflexifs associés uniquement aux occurrences de on, on notera que ce n'est pas du tout le cas avec (16), à la fois pour ce qui concerne le réflexif que pour ce qui concerne la conjugaison à la première personne du pluriel.

(16) Ce fut de justesse qu'on ne tua pas. On leur crevait les pneus, on les moquait, on les suivait, on s'amusait de cette guerre qui leur disputait le terrain. Sincèrement, nous nous amusions beaucoup (Frantext, R283, page 143)

(17) On se promet que si ça ne sert à rien, si on se trompe, nous n'en parlerons pas (Frantext, R175, page 89)

(18) Les autres jours nous allons chacun de notre côté, on ne se touche pas (Juste la fin $d u$ monde, page 56)

Les grammaires classiques indiquent qu'une même phrase ne devrait pas contenir plusieurs on désignant des référents différents, mais Le bon usage donne, aussitôt après ce constat, deux contre-exemples, dont (19), et nous en trouvons une multitude dans Frantext (20). On ne présente donc a priori pas de contraintes quant à sa coréférence. (Blanche-Benveniste, 2003) a fait de l'énoncé oral (21), prononcé par une employée de banque à une cliente qui lui 
demande à quelle date son chèque va être encaissé, l'exemple accrocheur de son analyse de on. (Béguelin, 2012) voit dans l'occurrence simultanée de plusieurs on non coréférentiels dans la même phrase une illustration de la capacité de on à désigner n'importe quel ensemble d'êtres animés, du fait de son extension vague. De fait, non seulement on peut désigner des référents divers de manière rapide et rapprochée, mais, de plus, ces variations référentielles ne semblent pas poser de problèmes de compréhension particuliers : « il est remarquable que on puisse changer de référence, d'une construction verbale à l'autre, sans que ce changement paraisse gênant, et sans même qu'il soit remarqué » (Blanche-Benveniste, 1987, page 23). Ces remarques, qui vont dans le sens de la compétence linguistique des interlocuteurs, vont aussi dans le sens de l'importance du flou: lors d'une interprétation immédiate, un lecteur ou interlocuteur ne cherche pas forcément à analyser dans les détails les possibilités référentielles - quel groupe de référents, avec quelles limites - de on, tout en détectant de manière immédiate quelles occurrences de on sont coréférentielles et lesquelles ne le sont pas. Une analyse possiblement partielle constitue aussi une facette de la sémantique de on.

(19) On vint dire à Mme de Kerkaradec qu'elle était servie, et l'on passa dans la salle à manger (Théophile Gautier, exemple cité par Grevisse \& Goosse, 2002, page 965)

(20) On raconte qu'on lui en a offert des sommes folles (Frantext, R284, page 100)

(21) On le renvoie comme ça et puis on nous le renvoie comme ça (BlancheBenveniste, 2003, page 43)

\subsection{Hypothèses et analyses de on}

A l'instar du pronom personnel de première personne, on peut désigner plusieurs référents possibles : narrateur zéro ; narrateur témoin ; personnage de l'histoire ; personnage générique, dans le cas de textes narratifs - auteur-écrivain; auteur-chercheur; auteur-argumentateur; auteur-générique, dans le cas d'écrits scientifiques (Carter-Thomas \& Chambers, 2012) auteur; auteur avec lecteur; auteur avec communauté limitée ; auteur avec communauté étendue ; lecteur ; autres, également pour les textes scientifiques (Fløttum et al., 2007) ipséité et altérité (Détrie, 1998) ; etc. Les classifications des valeurs de on sont nombreuses, cf. aussi (Anscombre, 2005) avec l'exemple des proverbes, ou encore (Malrieu, 2007) pour une étude sur plusieurs genres textuels.

Au-delà d'une question de référence, c'est tout le problème du point de vue qui est en jeu et que on contribue à rendre complexe. A partir d'un fragment - le récit d'un repas - de L'assommoir d'Emile Zola (22), Dominique Maingueneau en fait le pivot de sa notion d' « angélisme narratif » : " au lieu de chercher à attribuer ce fragment à telle ou telle instance ou mélange d'instances, on considérerait qu'on se trouve en fait au-delà ou en deçà de telles distinctions, dans une zone d'excès où les analyses classiques n'ont plus prise. Tout se passe comme s'il se produisait ici une sorte de trou interprétatif par un brouillage entre trois plans : celui du narrateur zéro, celui du narrateur-témoin (qui reste extérieur à l'histoire, même s'il participe du monde évoqué) et celui des personnages de l'histoire qui participent au repas » (Maingueneau, 2000, page 85). Il n'y a qu'un pas entre cette notion d'angélisme narratif dont le nom est dû aux propriétés d'un ange d'être porteur de message et d'intervenir de manière brève et imprévue - et la notion de groupe flou adoptée dans le projet MC4. On regroupe de manière floue plusieurs personnes, si tant est que l'on distingue plusieurs personnes possibles, d'une part dans le narrateur, d'autre part dans son auditoire. Nous avons vu qu'une communauté de chercheurs pouvait être incluse dans l'interprétation de on dans des écrits scientifiques. Dans le texte narratif, Alain Rabatel inclut pour sa part le lecteur, dans «le cas, fréquent, où on est le sujet d'authentiques perceptions représentées, en l'absence de tout focalisateur-personnage saillant: dans cette situation, on est un indéfini coréférant au 
narrateur anonyme comme au lecteur, invité à partager la position d'un observateur anonyme » (Rabatel, 2001, page 31). Le on qui se substitue au pronom de première personne ne vaut donc pas pour la première personne, et c'est d'ailleurs ce qui justifie son emploi plutôt que celui de je: il réfère à un groupe flou, qui inclut non seulement le narrateur avec ses diverses facettes, mais aussi le lecteur, cette inclusion elle-même étant floue.

(22) Ah ! nom de Dieu ! oui, on s'en flanqua une bosse! Quand on y est, on y est, n'estce pas, et si l'on ne se paie qu'un gueuleton par-ci par-là, on serait joliment godiche de ne pas s'en fourrer jusqu'aux oreilles. Vrai, on voyait les bedons se gonfler à mesure. Les dames étaient grosses. Ils pétaient dans leur peau, les sacrés goinfres ! La bouche ouverte, le menton barbouillé de graisse, ils avaient des faces pareilles à des derrières, et si rouges, qu'on aurait dit des derrières de gens riches crevant de prospérité (extrait entre deux passages narratifs dépourvus de on, cf. Maingueneau, 2000)

De même, on peut se substituer à l'interlocuteur, sans pour autant prendre la valeur du pronom de deuxième personne, au singulier (23) comme au pluriel (24) - exemple que l'on peut aussi interpréter comme un générique, ce qui contredit l'interprétation de (Oukada, 1982). Qu'il s'agisse d'affectation, de modestie, de délicatesse, d'ironie, ou encore d'une volonté de ne pas impliquer directement son interlocuteur (Sandfeld, 1970, page 335), c'est un phénomène qui semble plus fréquent dans des modes de communication médiatisés (forums sur internet, cf. Williams \& van Compernolle, 2009) qu'à l'oral ou à l'écrit plus classique.

(23) Tiens, on est beau aujourd'hui ! (exemple retenu par Oukada, 1982, page 97)

(24) Quand on est inquiet rien ne peut vous distraire (idem)

De fait, on peut prendre la valeur de n'importe quel pronom personnel, et, plus que cela, on peut référer à n'importe quel complexe de personne. C'est ce qui amène certains auteurs à laisser de côté la classification en première, deuxième et troisième personne, mais à parler plutôt, à la suite de Gustave Guillaume (cf. Blanche-Benveniste, 1987, page 29 ; BlancheBenveniste, 2003, page 47), de quatrième personne, de cinquième personne et de personne complexe.

C'est également ce qui amène d'autres auteurs à parler de valeur anaphorique pour on, cf. par exemple la classification de Catherine Viollet en : 1. valeur « déictique » (catégorie personne) ; 2. valeur " anaphorique » (catégorie personne); 3. valeur "parcours » (catégorie non-personne), où on vaut pour les humains en général et représente par exemple l'opinion publique ; 4. valeur « indécidable », qui relève du contexte au sens large (Viollet, 1988, page 69).

C'est enfin ce qui conduit à se rabattre sur la notion de contexte. Au tout début de sa thèse, Anje Müller Gjesdal note que : «si la référence est souvent mise en avant comme un critère fondamental dans l'attribution de sens aux pronoms personnels, nous pouvons affirmer que ce critère est insuffisant pour l'analyse de on. Il semble plutôt que le sens de on soit attribué à partir d'une interaction entre des éléments inhérents et des facteurs contextuels » (Gjesdal, 2008, page 12).

Nous ne pouvons qu'aller dans ce sens : pour nous, les pronoms sont des instructions, qui s'appliquent en contexte. Quand le contexte fait intervenir un narrateur dont les rôles sont flous - de par l'utilisation de verbes de perception, de par la façon dont la narration est rapportée, de par un procédé stylistique intentionnel, etc. - nous aboutissons à un on référant à un groupe flou, parce que le contexte est lui-même flou. Quand le contexte fait intervenir un 
référent (même pluriel) clairement délimité - par exemple lors d'une alternance de nous et de on manifestement coréférentiels - nous aboutissons à un on référant à un groupe strict, non flou, parce que le contexte n'est pas flou. Cette approche va à l'encontre de celle de (Schapira, 2006, page 509) qui - sur un sous-ensemble du problème, certes - consiste à rendre compte de la diversité des références possibles en postulant plusieurs on en français (pronom personnel générique et pronom de troisième personne indéfini, en l'occurrence), c'est-à-dire en statuant que on est polysémique. Avec notre notion de groupe flou, nous proposons d'éviter ce postulat avec un point de vue différent et potentiellement complémentaire.

\section{La référence de on : étude de corpus}

Dans le cadre du projet MC4, nous avons étudié les occurrences de on dans le corpus retenu par le projet. Comme la taille de celui-ci est modeste, peu d'exemples sont sortis. Nous avons alors choisi d'une part d'étudier de manière approfondie l'ensemble des occurrences de on dans la nouvelle L'occupation des sols de Jean Echenoz (extrait de MC4 présentant le plus grand nombre de phénomènes intéressants), et d'autre part de compléter nos observations dans des corpus supplémentaires. Nous avons ainsi effectué quelques requêtes dans la base Frantext - par exemple " on \&q(1,3) on » (on suivi de 1 à 3 mots suivis de on) pour extraire quelques exemples de cooccurrences rapprochées de on, et "on $\& q(1,4)$ nous », ce qui a permis d'obtenir l'exemple (17) et des exemples similaires - et étudié la pièce de théâtre Juste la fin du monde de Jean-Luc Lagarce, texte que nous avions étudié dans le cadre d'un projet parallèle et qui présente lui aussi un grand nombre de phénomènes intéressants. Nous nous focalisons sur des exemples illustrant la notion de référence floue, et nous distinguons ainsi, à titre indicatif, quatre sous-catégories : référence floue inclusive, référence floue exclusive, référence floue générique et référence totalement floue.

\subsection{Référence floue inclusive}

Une première utilisation de on, la plus réduite possible au sens référentiel, se rapproche de celle de je. C'est le cas dans (25), énoncé par la mère dans Juste la fin du monde. C'est aussi le cas dans (26) et (27), de manière plus floue car interférant avec une possible interprétation générique. Ce dernier exemple vient après une longue alternance de on et de je, dans laquelle la référence de on glisse petit à petit d'un générique à une quasi-coréférence avec je. Faut-il voir là un trait de caractère du locuteur (Louis) : égocentrisme ; perte de son identité ; besoin de se dire que son destin est celui de tout le monde? Faut-il y voir un jeu de Jean-Luc Lagarce ? Ce qui est sûr, c'est que on est un marqueur de référence suffisamment souple pour pouvoir être alterné avec un autre pronom personnel, même au singulier, sans casser une possible coréférence. Ce qui est sûr également, c'est que l'emploi de on ne vaut pas exactement celui de je, la répétition « on est bien » / « je suis bien » étant d'ailleurs là pour le prouver. On en déduit que dans ces trois exemples, les éléments qui apparaissent en gras forment des chaînes de référence, mais que la relation de coréférence entre on et je n'est pas exacte : on réfère au locuteur comme je, mais en ajoutant un flou que je ne communique pas. La relation de coréférence existe, seulement elle est floue. Dans cette mesure, on considère bien qu'il y a coréférence locale, c'est-à-dire qu'on va dans le sens de notre hypothèse postulant qu'au niveau intra-phrastique (ou intra-énoncé), la référence de on dépend des autres références présentes dans la phrase en privilégiant la coréférence locale. Bien qu'aucun on ne soit impliqué, cette hypothèse va aussi dans le sens de regrouper tout le monde et les enfants dans une même chaîne de référence en (25). 
(25) Je vous ennuie, j'ennuie tout le monde avec ça, les enfants, on croit être intéressante (Juste la fin du monde, page 14)

(26) On se dit ça, on se prépare (page 21)

(27) On pleure. On est bien. Je suis bien (page 44)

Une autre utilisation de on correspond à celle de nous, c'est-à-dire à la mention d'un groupe qui inclut le locuteur, que l'inclusion soit explicitée (28) ou qu'elle se déduise relativement facilement de la phrase ou du contexte (29). La coréférence entre nous et on est alors possible. Elle est même relativement fréquente dans le texte, comme on peut le voir avec plusieurs exemples dont (30). Dans le cadre de cette étude centrée sur les phénomènes de référence floue, nous noterons que seul l'exemple (29) est à retenir comme faisant partie de la catégorie « référence floue inclusive ».

(28) On dormait un peu, leur père et moi, sur la couverture (page 28)

(29) On travaillait, leur père travaillait, je travaillais (page 25)

(30) Nous sommes toutes les trois, comme absentes, on les regarde (page 69)

\subsection{Référence floue exclusive}

Les exemples où on a une référence floue tout en excluant le locuteur sont beaucoup plus rares dans notre corpus. Quand c'est le cas, comme dans (31), c'est que la phrase contient une occurrence de la première personne. Autrement dit, notre corpus vérifie la règle de schizophrénie partielle de (Blanche-Benveniste, 1987), du moins pour les occurrences de on qui ne conduisent pas à une référence trop floue, comme c'était le cas dans l'exemple (14) discuté plus haut.

(31) Et on renonce à moi, ils renoncèrent à moi (page 30)

\subsection{Référence floue générique}

L'exemple (32) fait apparaître une utilisation plus proche du générique que d'une référence à la famille. C'est un emploi fréquent dans Juste la fin du monde, et on l'a d'ailleurs déjà rencontré : dans (31) qui comprend une sorte de répétition - exactement comme dans (27) - le passage de on à ils marque un glissement du générique au spécifique, de manière à insister sur la responsabilité des individus spécifiques. Ce glissement est confirmé par un changement de temps, le on générique allant avec un verbe au présent et le ils spécifique avec un verbe au passé. Dans son emploi générique, on peut devenir quasiment impersonnel. C'est le sens de la répétition de (33) et de la forme presque figée de (34) qui apparaît aussi en (35) avec un autre on, qui, lui, réfère spécifiquement à Louis et Antoine. Ce dernier exemple, qui regroupe en une seule phrase deux référents différents pour la même forme, est un cas particulier si on considère qu'une des deux formes est figée (à rapprocher des on aurait dit et on ne sait où discutés plus haut), et ne contredit donc pas notre hypothèse de coréférence locale, intraphrastique ou intra-énoncé. Celle-ci est d'ailleurs illustrée par (36) : dans on klaxonnait, c'est probablement le père qui fait l'action. Pour les autres occurrences de on, toute la famille semble impliquée. Plutôt que de considérer deux référents différents, on en vient à interpréter on klaxonnait comme une action assumée par toute la famille - qui en devient le référent -, le père ne jouant qu'un rôle d'instrument. On est en quelque sorte inclusif : dès qu'il le peut, il tend à tout inclure. Par ailleurs, cet exemple qui comporte cinq occurrences de on souligne un aspect important de la narration : une série d'événements est rapportée, en une seule phrase, de manière groupée. L'utilisation systématique de on comme sujet permet de ne pas rompre la 
série d'événements, ce qui aurait été le cas avec des références précises - la famille pour certains événements, le père seulement pour le coup de klaxon. D'autant plus qu'une mention du père au milieu de plusieurs mentions de la famille aurait eu pour effet d'augmenter la saillance du père, la rupture dans une continuité étant un facteur de saillance important. Or le but ici n'était probablement pas de mettre en avant la saillance des personnages, mais, bien au contraire, de mettre en avant la saillance des événements. C'est un point sur lequel nous reviendrons dans la section 4. Pour l'instant, nous noterons que les cinq occurrences de on forment une seule chaîne de référence, avec un flou sur le on de on klaxonnait. En tout cas, l'hypothèse de la coréférence locale est vérifiée.

(32) On ne peut pas plaisanter (page 16)

(33) Et alors il faut te chercher, on doit te chercher (page 59)

(34) Comment est-ce qu'on dit? « d'une pierre deux coups » (page 64)

(35) On ne devrait jamais se lâcher, serrer les coudes, comment est-ce qu'on dit? (page 68)

(36) On disait qu'on «partait en vacances », on klaxonnait, et le soir, en rentrant, on disait que tout compte fait, on était mieux à la maison (page 28)

\subsection{Référence totalement floue}

Dans l'exemple (37), crié par Suzanne en réaction à l'appel «Louis !» lancé par la mère, la référence de on va au-delà des règles simples. Au minimum, elle devrait inclure Louis et Suzanne. Or la scène met en présence Suzanne et Antoine ! Dans ce cas, on ne peut pas valoir pour $i l$, dans la mesure où Louis n'est justement pas là. Mais on ne peut pas valoir non plus ni pour je, ni pour nous, ni pour vous, ni pour ils. La référence ne peut pas non plus être étendue à un collectif tel que les enfants, puisque les enfants ne sont pas rassemblés et ne vérifient pas la propriété d'être là. Au final, on aboutit à une référence brouillée, perturbante, probablement pour entretenir les incompréhensions et les problèmes de communication des scènes précédentes.

\section{(37) Oui ? On est là ! (page 57)}

«On renvoie toujours à un ensemble de personnes, conçu non comme l'addition de personnalités distinctes, mais comme une masse indénombrable » (Leeman, 1991, page 108). C'est ce que montrent certains des exemples ci-dessus, et notamment l'exemple (37), où il est effectivement impossible de procéder à l'addition de référents distincts. C'est pourquoi nous avons exploité la notion de groupe flou, ou celle qui lui est liée de relation de coréférence floue, ou encore celle qui les récapitule en un seul terme : référence floue.

$\mathrm{Au}$ final, suite à l'étude de l'ensemble des occurrences de on dans Juste la fin $d u$ monde, nous vérifions nos deux hypothèses : on tend à référer de manière privilégié à un groupe flou, avec une légère tendance à inclure le maximum de personnes. Cette tendance est d'ailleurs frappante sur un point : on a parfois l'impression que le groupe flou inclut le lecteur (ou auditeur), c'est-à-dire qu'il tend à impliquer celui-ci dans ce qui est dit, comme le remarquait Alain Rabatel (cf. section 2.3). Avec on, ce n'est plus tant le référent que l'événement qui est mis en avant par le locuteur. Cela rejoint les études de proverbes (Anscombre, 2005).

Par ailleurs, cela rejoint également notre étude de L'occupation des sols de Jean Echenoz: dans (38), on réfère a priori au groupe constitué par le père Fabre et le fils Paul, groupe construit dès la première phrase du texte. Sauf qu'au fil du texte, la référence devient de moins en moins claire (le texte contient 25 occurrences de on, à mettre en rapport avec sa 
longueur modeste). Les liens avec les référents sont de plus en plus coupés, et on ne retient au bout d'un moment que les événements qui sont narrés (39). Dans ces deux exemples qui sont essentiels dans le récit, le verbe compte plus que son sujet. Cette remarque se rapproche de la notion de structure verbale, utilisée par Pierre Attal (Danon-Boileau \& Morel, 1994) - par opposition avec la structure à thème-propos, la structure quantifiante et les structures génériques, et par comparaison avec le passif impersonnel du latin - pour analyser un exemple comme " on crie à côté » par «il y a des cris à côté ». Cette remarque rejoint celle faite à la fin de la section 3.3, et nous y reviendrons dans la section 4 .

(38) On gratte, on gratte et puis très vite on respire mal, on sue, il se met à faire terriblement chaud.

(39) Regarde un peu le soleil qu'on a.

Dans le texte de Jean Echenoz comme dans celui de Jean-Luc Lagarce, les emplois de on contribuent à entretenir du flou dans les références. C'est d'ailleurs l'un des aspects majeurs de L'occupation des sols, texte qui par ailleurs fait intervenir un référent particulier : la mère («elle ») qui se confond avec une représentation d'elle-même peinte sur un mur (« elle » également).

\section{La coréférence de on et ses rôles discursifs}

\subsection{Plusieurs degrés de coréférence}

La référence de on est vague : elle peut être générique, elle peut être spécifique avec plusieurs interprétations possibles, et surtout du flou qui peut persister tout au long d'un texte. La coréférence de on est vague : à partir du moment où la référence est floue, il est difficile de construire des relations de coréférence strictes quand un on est impliqué. On en vient à s'interroger sur ces relations de coréférence et sur le rôle des chaînes de référence dans un texte. Nous avons déjà évoqué la possibilité d'une relation de coréférence floue. Compte tenu du flou sur les personnes regroupées par on, nous proposons de distinguer plusieurs degrés de coréférence :

- Degré 1 : «coréférence stricte ». Les référents sont identiques, qu'il s'agisse d'un individu ou d'un groupe. C'est le cas dans les exemples où on n'apparait pas (une suite de nous clairement coréférentiels), dans les exemples où on est explicité et où sa référence est donc non seulement stricte, mais correspond de plus à d'autres expressions référentielles dans le texte (28), et dans les exemples où plusieurs occurrences de on réfèrent strictement aux mêmes personnes, comme en (26) ou en (38).

- Degré 2: «coréférence inclusive ». L'un des référents - de type "groupe flou »inclut l'autre. C'est le cas dans les exemples d'alternances de on et d'un autre pronom personnel, le fait que les marqueurs diffèrent signalant un léger flou autour de la référence de on et donc de la chaîne de référence. Les exemples (25) et (27) font partie de cette catégorie. C'est le cas également quand on est en présence de plusieurs on, la référence de certains incluant celle des autres. L'exemple (36) avec on klaxonnait fait partie de cette catégorie.

- Degré 3: «coréférence floue ». Les deux référents sont des groupes flous et l'intersection entre les deux est possible, tout en restant potentiellement floue ellemême. C'est le cas des exemples (22) et (31). 
On intervient donc d'une manière particulière dans les chaînes de référence d'un texte : là où des expressions référentielles définies et des pronoms personnels opèrent essentiellement au degré 1 , on peut intervenir dans les degrés 1,2 et 3. C'est le marqueur privilégié du degré 3 , avec ça pour ce qui concerne la référence aux événements (Landragin, 2007). Nous noterons que distinguer trois degrés permet de maximiser les liens de coréférence intra-phrastiques et extra-phrastiques. Avec les degrés 2 et 3, on tend à maximiser les liens entre phrases et donc la cohérence et la cohésion d'un texte.

Par ailleurs, nous avons vu que l'interprétation d'une succession de on peut varier dans la même phrase. Dans les exemples (19), (20) et (21), les occurrences de on ne permettent de construire entre elles aucun lien de coréférence, quel que soit le degré considéré : il n'y a pas d'intersection entre les référents, qui d'ailleurs ne sont probablement pas du type "groupe flou ». A première vue, ces exemples ne vérifient pas notre hypothèse favorisant la coréférence locale. Il est à noter cependant que toute coréférence n'en est pas absente : chacune des occurrences de on appartient à une chaîne de référence. Celle qui comprend le premier on de l'exemple (21) comprend également l'occurrence de nous. Pour les autres exemples, le contexte gauche et le contexte droit devraient permettre sans aucun doute de faire apparaître des expressions coréférentielles. Pour l'exemple (20), c'est d'ailleurs le cas (on le vérifie facilement dans Frantext). Autrement dit, ces exemples ne vérifient pas notre hypothèse au niveau de la coréférence de plusieurs on, mais vérifient probablement notre hypothèse de coréférence locale, pour peu que l'on tienne compte de l'ensemble des référents impliqués et que l'on dépasse quelque peu le contexte de la phrase.

\subsection{On marqueur de cohésion et de cohérence}

Finalement, on ne serait-il pas plus lié à des phénomènes discursifs qu'à des phénomènes référentiels ? C'est dans ce sens que va notre appréhension de la coréférence, notamment avec le troisième degré, et c'est aussi ce que nous montrent nos exemples : en maintenant - même de manière floue - des chaînes de référence, en alternant facilement avec d'autres formes de référence, en englobant le maximum de référents, on possède des propriétés essentielles d'un marqueur de cohésion et de cohérence.

Plusieurs auteurs ont déjà souligné le peu d'importance de la référence de certains on et l'importance en conséquence de la construction du discours. Nous avons déjà cité les travaux de Claire Blanche-Benveniste, ou encore la structure verbale de Pierre Attal. De même, Josiane Boutet insiste sur la part de l'indécidabilité et de l'indétermination dans l'interprétation de on, ce qui l'amène à analyser « on embauche dans la boîte " par « il y a de l'embauche dans la boîte » (Boutet, 1986, page 44), ou encore « on devrait faire de la musique là-dedans » par «il devrait y avoir de la musique là-dedans », du moins dans l'une des interprétations possibles - les autres étant référentielles (inclusive et exclusive). Dans un contexte différent mais un même ordre d'idée, (Koenig \& Mauner, 1999) proposent que on incarne un troisième type de syntagme nominal, ni défini ni indéfini, mais "a-definite », nouvelle catégorie qu'ils considèrent comme un équivalent lexical d'un argument implicite (dans la mesure où il sature une position argumentale sans être accessible à la reprise anaphorique). La modélisation qui en résulte se place dans la Théorie de Représentation du Discours, mais (Prince, 2006) en propose une comparable dans le cadre de la Théorie du Centrage (cf. aussi Cabredo Hofherr, 2008 ; Creissels, 2011).

C'est en suivant cette voie que l'on peut revenir sur des exemples tels que (8) : si « on m'a volé mon vélo deux fois » diffère de «quelqu'un m'a volé mon vélo deux fois », c'est parce que la phrase avec on vaut pour «mon vélo a été volé deux fois ». C'est aussi en suivant cette voie que l'on peut trouver une interprétation rationnelle à l'exemple (37) : dans « on est 
là », nous avons vu qu'aucun personnage ni groupe de personnes, strict ou flou, ne pouvait être considéré comme le référent de on. Il ne reste plus qu'une solution : considérer que dans ce cas, on joue le rôle de référent minimal (quasiment non instancié), c'est-à-dire considérer que l'énoncé vaut plus pour «être là » que pour quelqu'un en particulier. Comme pour le grattage, comme pour «il y a des cris à côté », comme pour la succession des événements dans (36), le texte - grâce à l'utilisation de on - ne met plus en avant des entités de discours de type référents humains, mais rend saillants des événements. Dans (36), c'est la succession « partir en vacances - klaxonner - rentrer - dire qu'être mieux à la maison » qui importe, et non la succession de références à la famille et/ou au père de famille.

Enfin, nous reprendrons pour finir le fragment tiré de L'assommoir décrivant un banquet donné par Gervaise (22) : sept occurrences de on apparaissent dans ce fragment, avec comme référence possible celle d'un personnage générique, qui ne se réduit pas à un personnage particulier (déjà bien repéré par le lecteur), qui ne peut pas être un narrateur extérieur (car le style du texte ressemble à ce que dirait quelqu'un appartenant à l'entourage des personnages) et que Dominique Maingueneau explicite en tant que «n'importe lequel des convives ", tout en notant que ce personnage générique joue aussi le rôle de narrateur (Maingueneau, 2000, page 76). Par ailleurs, les deux on de "quand on y est, on y est » peuvent s'interpréter comme plus génériques encore, de même que « on serait joliment godiche de ne pas s'en fourrer jusqu'aux oreilles ». Autrement dit, il est possible de trouver des nuances référentielles à peu près partout dans ce fragment. La notion de groupe flou permet de modéliser l'ensemble en tant qu'une seule chaîne de référence, avec divers degrés de flou, par exemple pour rendre compte de la plus grande généricité de « quand on y est, on y est », ou encore pour rendre compte de la présence potentielle d'un narrateur voire du lecteur dans le groupe de référents.

\section{Conclusion}

En multipliant les notions et les hypothèses autour du problème de la référence de on personne complexe, complexe de personnes différentes, narrateur témoin, membre quelconque d'une collectivité, narrateur angélique, schizophrénie partielle, ipséité, a-definite, énallage de personne, etc. - il apparaît que la notion de référence floue s'avère pour le moins dans la lignée des travaux existants. La référence floue permet d'une part de rendre compte de la multiplicité des cas de référence de on dans un cadre unique, et d'autre part de mettre en avant le fait que résoudre la référence n'est pas forcément une priorité absolue lors de l'interprétation de on. L'identification exacte des référents peut rester une question ouverte, non seulement localement, mais aussi plus durablement : le lecteur ou l'interlocuteur n'est pas gêné dans sa compréhension, même s'il n'arrive pas à attribuer des référents exacts et sûrs à toutes les occurrences de $o n$.

La référence floue fait écho à l'indétermination et à la sous-détermination. Pour la prendre en compte dans notre modélisation des chaînes de référence, il nous a fallu distinguer plusieurs degrés de participation d'une expression référentielle à une chaîne. Ce principe, lui, fait écho à celui de la distinction entre « maillon fort » et « maillon faible » (Landragin, 2011 ; Schnedecker \& Landragin, ce volume). Il permet de relâcher des contraintes d'appartenance trop strictes d'un référent à une chaîne de référence, au prix cependant d'une modélisation plus complexe, aussi bien d'un point de vue linguistique que d'un point de vue informatique. Les outils d'annotation manuelle comme ANALEC (Mélanie-Becquet \& Landragin, ce volume) permettent heureusement de gérer de tels modèles, même si nous ne l'avons pas fait dans le cadre du projet MC4. Ce point constitue notre principale perspective à ce travail. 
Il ne nous semble pas forcément pertinent de chercher à explorer plus profondément la nature du flou : à trop vouloir caractériser le flou, on lui fait perdre sa nature, on tend à caractériser quelque chose qui est flou non seulement pour le lecteur, mais aussi - en partie peut-être - pour l'auteur. Nous proposons, dans les cas concernés, de laisser inachevée l'identification des référents, et, par conséquent, de diminuer l'importance de l'acte de référence par rapport à l'acte de prédication. Cela fonctionne très bien pour le banquet de L'assommoir analysé par (Maingueneau, 2000), pour l'interprétation des relations entre les personnages de Juste la fin du monde, et pour le grattage final de L'occupation des sols. Ce sont les événements qui comptent avant tout, et non les participants. La cohérence et la cohésion du texte sont assurées par la succession des événements et par les chaînes de on, les personnages n'étant que secondaires dans ce mécanisme discursif. De ce point découle également une perspective de recherche: celle consistant à explorer de manière plus approfondie les liens entre notre modélisation et celle des approches "good-enough 》 de l'interprétation du langage.

\section{Remerciements}

Les auteurs remercient Michel Charolles pour ses encouragements et pour les discussions autour d'une version préliminaire de cette étude, Patricia Cabredo Hofherr pour ses conseils bibliographiques, et les relecteurs anonymes pour leurs analyses et conseils profitables.

\section{Références bibliographiques}

ANSCOMBRE C. (2005) «Le On locuteur : une entité aux multiples visages », In : Bres J. et al. (Eds.) Dialogisme, polyphonie : approches linguistiques, De Boeck Duculot, pp. 75-94.

BEGUELIN M.-J. (2012) «La concurrence entre nous et on en français », Colloque "Noi - Nous Nosotros. Sur les traces d'un pronom », Université de Zürich.

BLANCHE-BENVENISTE C. (1987) "Le pronom on : propositions pour une analyse », in : Chevalier J.C. \& Delport M.-F. (Dir.) Mélanges offerts à Maurice Molho, Linguistique - Volume III, Les Cahiers de Fontenay, pp. 15-30.

BLANCHE-BENVENISTE C. (2003) «Le double jeu du pronom on», in : Berré M. et al. (Eds.), La syntaxe raisonnée, De Boeck Supérieur « Champs linguistiques », pp. 41-56.

BOUTET J. (1986) «La référence à la personne en français parlé : le cas de 'on' », Langage et société 38 , pp. $19-50$.

CABREDO HOFHERR P. (2008) «Les pronoms impersonnels humains - syntaxe et interprétation », Modèles linguistiques XXIX-1, vol 57, pp. 35-56.

CARTER-THOMAS S. \& CHAMBERS A. (2012) «From text to corpus: A contrastive analysis of first person pronouns in economics article introductions in English and French », in : Boulton A. et al. (Eds.), Corpus-Informed Research and Learning in ESP, John Benjamins, pp. 17-39.

CHARAUdeAu P. (1992) Grammaire du sens et de l'expression, Hachette, Paris.

Creissels D. (2011) "Impersonal Pronouns and Coreference: two Case Studies », Workshop on Impersonal Pronouns, téléchargeable sur http://www.umr7023.cnrs.fr.

DanON-Boileau L. \& Morel M.-A. (Dir., 1994) L'indéfini, Faits de Langues 4, PUF, Paris.

DETRIE C. (1998) «Entre ipséité et altérité : statut énonciatif de «on» dans Sylvie », L'information grammaticale 76 , pp. 29-33.

DEULOFEU J. (1989) «Les couplages de constructions verbales en français parlé : effet de cohésion discursive ou syntaxe de l'énoncé », Recherches sur le français parlé 9, pp. 111-141.

FløtTUM K., JonASSON K. \& NoREN C. (2007) On : pronom à facettes, De Boeck Duculot « Champs Linguistiques », Bruxelles.

FRANTEXT, Base textuelle FRANTEXT, Analyse et Traitement Informatique de la Langue Française CNRS \& Université de Lorraine, http://www.frantext.fr. 
FuCHS C. (1996) Les ambiguïtés du français, Ophrys, Paris.

GJESDAL A.M. (2008) Etude sémantique du pronom on dans une perspective textuelle et contextuelle, $\mathrm{PhD}$ Thesis, University of Bergen.

Grevisse M. \& Goosse A. (2002) Le bon usage, $14^{e}$ édition, De Boeck Duculot, Bruxelles.

KOENIG J.-P. \& MAUNER G. (1999) «A-definites and the semantics of implicit arguments », Journal of Semantics 16(3), pp. 207-236.

LANDRAGIN F. (2007) «L'anaphore à antécédent flou : une caractérisation et ses conséquences sur l'annotation des relations anaphoriques ", Journée d'étude de l'Association pour le Traitement Automatique des Langues (ATALA) sur la résolution des anaphores, Paris.

LANDRAGIN F. (2011) «Une procédure d'analyse et d'annotation des chaînes de coréférence dans des textes écrits », Corpus 10, http://corpus.revues.org, pp. 61-80.

LEEMAN D. (1991) «On thème », Lingvisticae Investigationes 15, pp. 101-113.

MAINGUENEAU D. (2000) «Instances frontières et angélisme narratif », Langue française 128, pp. 7495.

MALRIEU D. (2007) «Contribution à une linguistique néo-saussurienne des genres de la parole (2) : analyse des valeurs d'indexicalité interlocutoire de on selon les genres textuels », Linx 56, pp. 157176.

OUKADA L. (1982) « On on », The French Review 56(1), pp. 93-105.

PRINCE E.F. (2006) «Impersonal pronouns in French and Yiddish: Semantic reference vs. discourse reference ", in: Birner B.J. \& Ward G. (Eds.) Drawing the Boundaries of Meaning, John Benjamins, pp. 295-315.

RABATEL A. (2001) «La valeur de on pronom indéfini / pronom personnel dans les perceptions représentées ", L'information grammaticale 88, pp. 28-32.

REY A. (Dir., 2012) Dictionnaire historique de la langue française, Dictionnaires Le Robert.

SANDFELD K. (1970) Syntaxe du français contemporain. I. Les pronoms, Honoré Champion, Paris.

SCHAPIRA C. (2006) «On pronom indéfini », in: Corblin F. et al. (Eds.), Indéfini et prédication, Presses de l'Université Paris-Sorbonne, Paris, pp. 507-518.

TLFI, Trésor de la langue française informatisé, Analyse et Traitement Informatique de la Langue Française, http://atilf.atilf.fr/tlf.htm.

ViOLleT C. (1988) « Mais qui est On ? », Linx 18, pp. 67-75.

Williams L., VAN COMPERNOlle R.A. (2009) «On versus $t u$ and vous: Pronouns with Indefinite Reference in Synchronous Electronic French Discourse », Language Sciences 31, pp. 409-427. 